\title{
Spontaneous Left Anterior Descending Coronary Artery Dissection in a Teenager
}

\author{
Camilla Torlasco, MD, ${ }^{\text {ab }}$ Simonetta Blengino, MD, ${ }^{\text {a }}$ Mauro Musmeci, MD, ${ }^{\text {a }}$ Gerardina Fratianni, MD, ${ }^{a}$ \\ Gabriella Malfatto, MD, PHD, ${ }^{\mathrm{a}}$ Gianfranco Parati, $\mathrm{MD}^{\mathrm{a}, \mathrm{b}}$
}

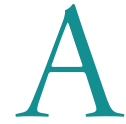

19-year-old man, heavy smoker, was admitted for subacute anterior ST-segment elevation myocardial infarction (Figure 1). He played a soccer match 48 hours earlier and had complained of severe chest pain ever since. Coronary angiography was suggestive for a dissection of the proximal-to-mid left anterior descending artery. Dissection flap, subocclusion of the first septal branch, and intramural thrombosis were noted (Figure $2 \mathrm{~A}$ and $2 \mathrm{~B}$, Online Video 1). Direct stenting (drug-eluting stent) resulted in Thrombolysis in Myocardial Infarction flow grade 3 (Figure 2C, Online Video 2). Cardiac magnetic resonance at 3 months showed moderate left ventricular function impairment, persistence of microvascular obstruction, extensive scar, and fatty metaplasia (Figure 3, Online Video 3). Screening for collagenopathy, autoimmune, and coagulation disorders was negative.

Spontaneous coronary artery dissection accounts for approximately $0.1 \%$ to $0.4 \%$ of all acute coronary syndromes. Typically affecting women, this condition rarely presents in young males, often following intense exercise (1). Intimal tear or bleeding from the vasa vasorum in predisposed arteries result in the separation of intima from media tunica (2). Conservative management to allow spontaneous healing is usually preferred, but some circumstances, including ongoing ischemia and recurrent chest pain, call for revascularization (3). Because of the nature of the disease, percutaneous coronary intervention is challenging (e.g., technical difficulties, risk of dissection or hematoma extension, side branch occlusion) and often unsuccessful (reported success rate, <50\%) (1). Overall prognosis is excellent, but a lifelong risk of recurrent spontaneous coronary artery dissection persists. In our patient, despite effective revascularization, late presentation led to severe left ventricular damage.

ADDRESS FOR CORRESPONDENCE: Dr. Camilla Torlasco, IRCCS Istituto Auxologico Italiano, Via Magnasco $n^{\circ}$ 2, 20149, Milan, Italy. E-mail: c.torlasco@ auxologico.it. 


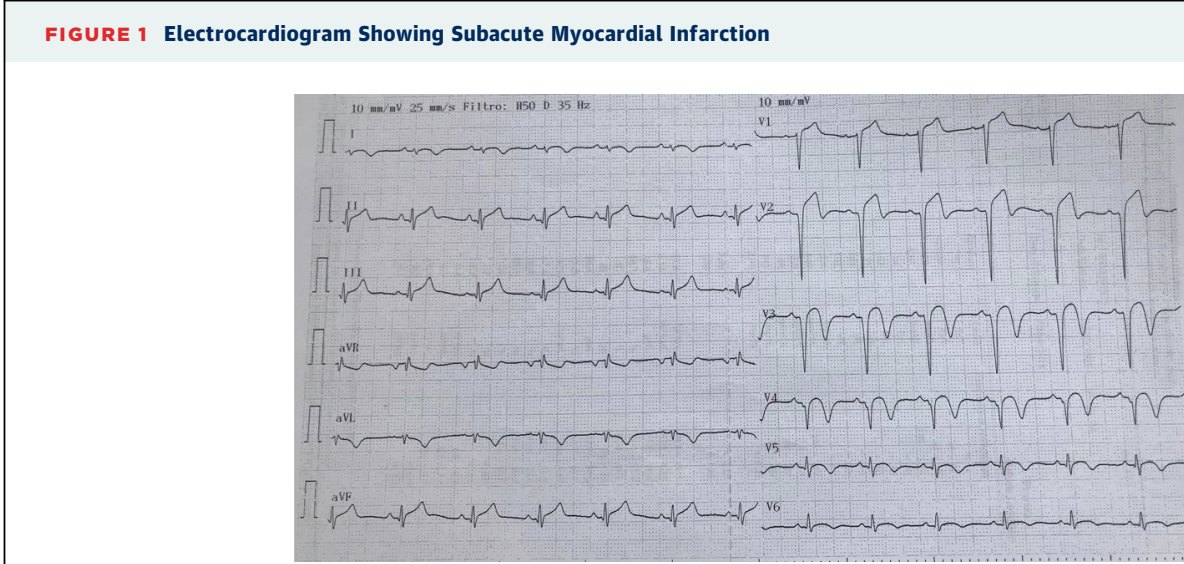

Anterior QS complex, ST-segment elevation, biphasic T waves.
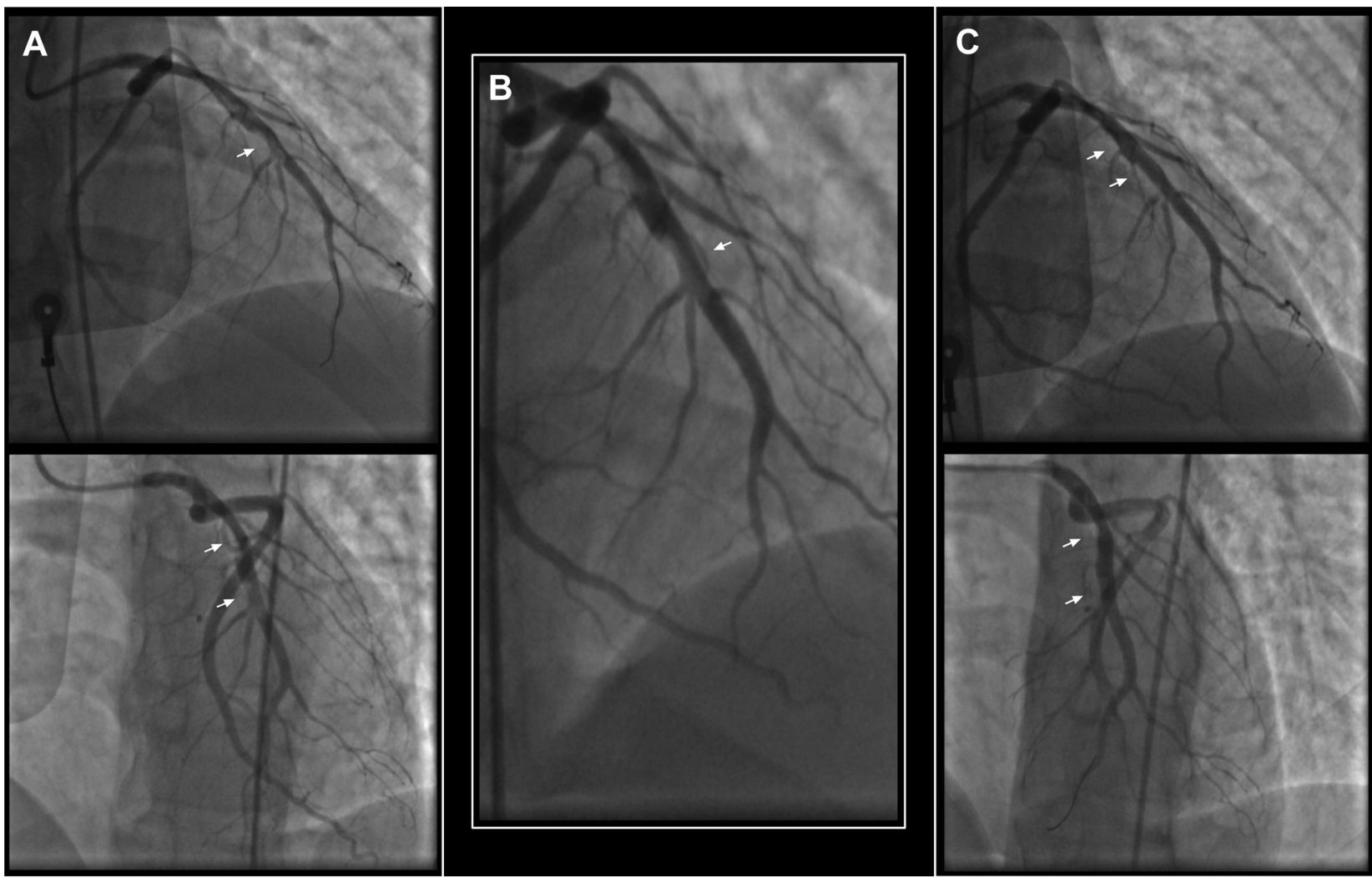

(A) Tubular long lesion with multiple intramural hematomas (white arrows), LAD flow TIMI flow grade 2 (Online Video 1). (B) Suspect dissection flap at the mid-LAD section (white arrow) (Online Video 1). (C) TIMI flow grade 3 flow after stenting, as highlighted by the white arrows (Online Video 2). LAD = left anterior descending; $\mathrm{TIMI}=$ Thrombolysis In Myocardial Infarction. 


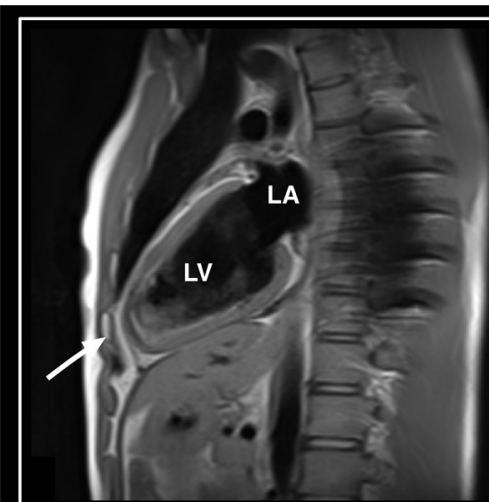

A

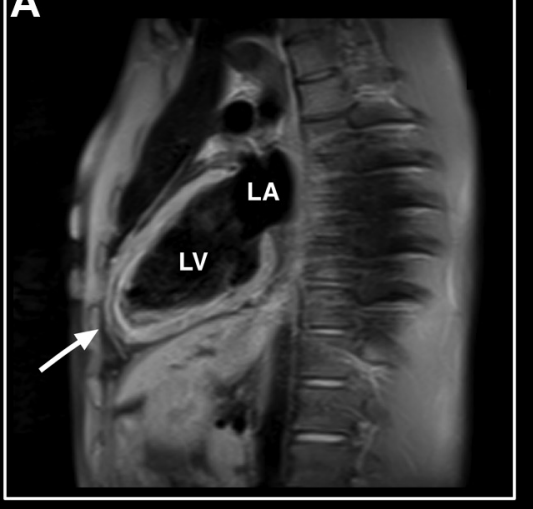

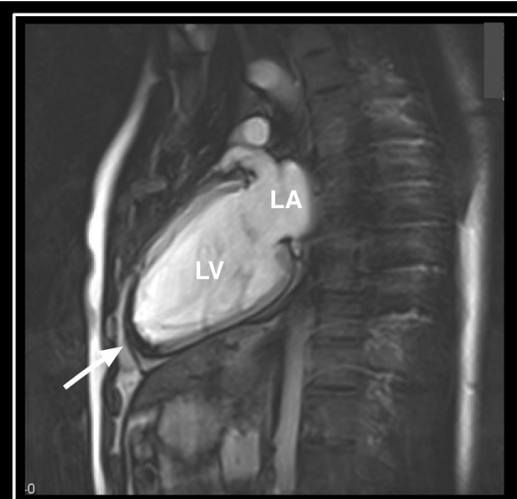

B

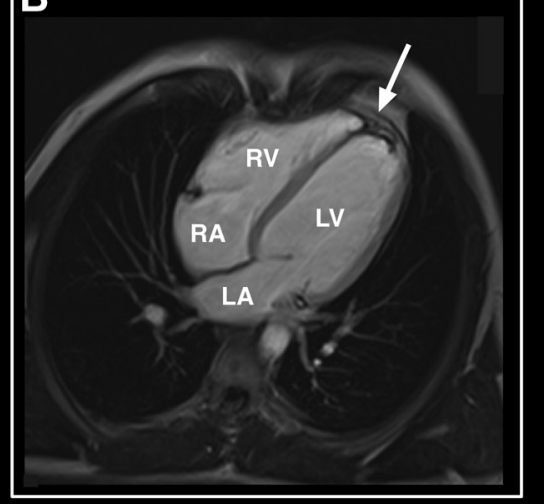

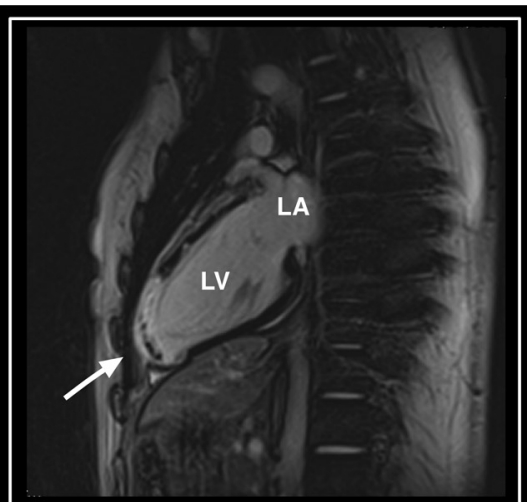

C

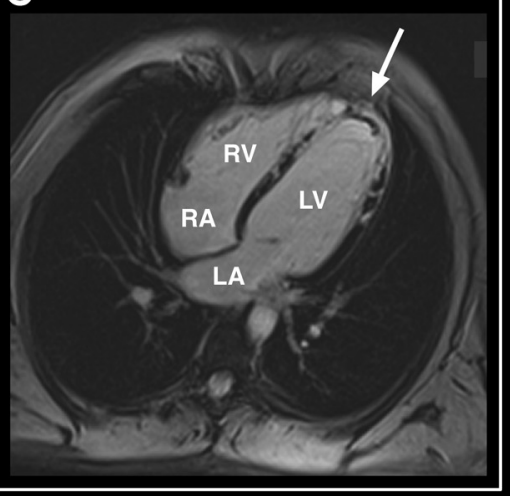

(A) Anteroapical fatty metaplasia at T1-weighted (with/without fat suppression) images (white arrow). (B) Persistence of anteroapical microvascular obstruction at early gadolinium enhancement images (white arrow). (C) Extensive ischemic late enhancement in the anteroseptal, inferior walls, and apex (white arrow). LA = left atrium; LV = left ventricle; RA = right atrium; RV = right ventricle. See Onlin 2 . 3.

\section{REFERENCES}

1. Aziz S. Spontaneous coronary artery dissection. E-Journal of Cardiology Practice [Internet]. 2018. Available at: https://www.escardio.org/Journals/ E-Journal-of-Cardiology-Practice/Volume-14/ spontaneous-coronary-artery-dissection. Accessed October 16, 2018.

2. Saw J, Aymong E, Sedlak T, et al. Spontaneous coronary artery dissection: association with predisposing arteriopathies and precipitating stressors and cardiovascular outcomes. Circ Cardiovasc Interv 2014;7:645-55.

3. Díez-Delhoyo F, Sanz-Ruiz $R$, SarnagoCebada F, et al. Spontaneous coronary artery dissection: failure of the conservative strategy due to predominance of the false lumen. J Am Coll Cardiol Intv 2017;10:e139-40.
KEY WORDS acute myocardial infarction, cardiac magnetic resonance, coronary artery dissection, coronary percutaneous intervention

9 A APPENDIX For supplemental videos, please see the online version of this paper. 